\title{
Retraction Note to: Profiling of differentially expressed microRNAs (miRNAs) during differentiation of rat hepatic oval cells (HOCs) into hepatocellular carcinoma (HCC) cells
}

\author{
R. H. Xu ${ }^{1,2} \cdot$ L. Y. Zheng ${ }^{3}$ D. L. $\mathrm{He}^{1} \cdot$ J. Meng ${ }^{1}$ L. P. Xia ${ }^{1}$. \\ X. B. $\mathrm{Hao}^{4}$ - Z. Z. Zhang ${ }^{1,2}$
}

Published online: 18 August 2015

(C) Federación de Sociedades Españolas de Oncología (FESEO) 2015

Retraction Note to: Clin Transl Oncol (2015)

17:230-237

DOI 10.1007/s12094-014-

1218-2

The Publisher and Editor retract this article in accordance with the recommendations of the Committee on Publication Ethics (COPE). After a thorough investigation we have strong reason to believe that the peer review process was compromised.

The online version of the original article can be found under doi:10.1007/s12094-014-1218-2.

X. B. Hao

XinbaoHaoxbh@163.com

$\triangle$ Z. Z. Zhang

ZhongzhiZhangzzz@163.com

1 Department of Oncology Surgery, The Affiliated Hospital of Hainan Medical College, Haikou 570102, China

2 Graduate School of China Medical University, No. 92 beierma Lu, Shenyang 110001, China

3 Department of Neurology, The Affiliated Hospital of Hainan Medical College, Haikou 570102, China

4 Department of Hematology/Oncology, The Affiliated Hospital of Hainan Medical College, No. 31 Longhua Lu, Haikou 570102, China 